\title{
CAUSAL AND GEOMETRIC RELATIONS *
}

\author{
Douglas $W$. Shrader, Jr.
}

Is space curved? The discovery of Non-Euclidean geometries inspired several attempts to determine empirically the topology of physical space. Hans Reichenbach recognized that a number of causal principles are presupposed in these experiments and suggested that. for geometry to be an empirical matter, we have to introduce the requirement that no causal relations are violated (that there are no causal anomalies). Though objectionable in certain respects, I think Reichenbach's thesis represents a significant insight into the interdependence of geometry and causation. The geometry we ascribe to physical space will help determine the nature of our causal laws, and vice-versa. But close as the connections between geometry and causation might be, they do not guarantee a unique prescription--even in conjunction with unlimited experience, a system of causal laws may not suffice to pick out a unique geometry, or vice-versa.

1. Introduction. Euclid developed his system of planar geometry sometime around 300 B.C. Centuries passed before anyone realized that other geometric systems were even mathematically possible. Today we have a well-accepted scientific theory (Einstein's General Theory of Relativity) which actually claims that space is curved (that a Non-Euclidean geometry applies).

But the choice of a geometry is not purely arbitraxy. Well over a hundred years ago Gauss devised an experiment to trace a triangle between three mountain peaks so as to determine whether or not the interior angles summed to a number different from 180. Since then there have been a number of other attempts to determine empirically the topology of physical space (i.e. to decide which geometry applieg).

*An earlier version of this paper was presented at the American Graduate student Conference on Philosophy, October 22, 1976, The American University, washington, D. C. 
Hans Reichenbach recognized that a number of causal principles are presupposed in these experiments and suggested that, for geometry to be an empirical matter, we have to introduce the requirement that no causal relations are violated (that there are no causal anomalies). only in this way, says Reichenbach, does the question about the topology of space even constitute a welldetermined question. 1

Though objectionable in certain respects, I think Reichenbach's thesis represents a significant insight into the interdependence of geometry and causation. In this paper therefore, I will undertake to defend the general drift of that thesis: there are indeed close connections between geometry and causation. The geometry we ascribe to physical space will help determine the nature of our causal laws, and vice-versa. But perhaps I am getting ahead of myself; for reasons which will become apparent later, before I can appropriately deal with this thesis I must first discuss Reichenbach's notion of a universal force.

2. Universal Forces. Universal forces, in contradistinction to differential forces, are ones (i) which affect all substances in exactly the same way and (ii) against which no insulating walls can be built ( $c$ f : Reichenbach, p. 13). Reichenbach further distinguishes between universal forces "preserving coincidences" and those "destroying coincidences." The forces preserving coincidences are constant throughout the entire universe and consequently neither the force nor its effects can be objectively determined. The ones destroying coincidences are active only in localized regions of space and therefore are demonstrable: Adolf Grünbaum [ Philosophical Probiems of Space and Time, 2nd ed. (Boston: Reidel, 1973)J has subsequently characterized those universal forces which preserve coincidences as "metaphorical" and those which destroy coincidences as "literal." It is these "literal" universal forces which will prove most important for our present concerns.

Reichenbach explains that, actually, "The distinction between universal and differential forces merely classifies the phenomenon as belonging in geometry or in physics" (Reichenbach, p. 27). A force is something which is responsible for a geometrical change; consequently the existence of a force is dependent upon the coordinate definition of geometry.

If our measurements indicated that a geometry G' obtained, we could still choose to call G the "actual" geometry and posit a universal force $F$ to explain the discrepancy in our measurements. Thus Reichenbach can 
assert, "We obtain a statement about physical reality only if in addition to the geometry $G$ of space its universal field of force $F$ is specified. Only the combination

$$
\mathbf{G}+\mathbf{F}
$$

is testable statement" (Reichenbach, p. 33). Simply being told that a geometry $G$ applies will not convey any information about physical space unless we also know what, if any, universal forces are being employed. 2

3. Causal Anomalies. Suppose we have specified the geometry and the universal field of force (i.e. we have a statement of the form $G+F)$; have we now adequately described the topology of physical space? Reichenbach says no; there still may be causal anomalies which we have not yet considered.

To illustrate, Reichenbach asks us to imagine a system of concentric spherical shells (figure I) and the experiences of a man climbing about on them (cf. Reichenbach, pp. 63-66). After measuring shel1. 1, the man proceeds to shell 2. Measuring 2, he finds it to be smaller than 1 and thus concludes that it is situated inside 1. He is astonished however to find 3 as large as 1 and hence larger than 2. In Euclidean geometry, 3 has to be smaller than 2 since it is enclosed by 2 . Our explorer chooses to adhere to Euclidean geometry and explains his measurements by a contraction of the measuring rod; his rod and also his body contract so that 3 appears larger than 2. In Reichenbach's terminology, the explorer posits a "universal force" to explain the difference between the "actual" and the "apparent" geometry.3 Going on to shell 4 he measures it to be even larger than $3 ;$ his rod has further contracted. 5 he again finds as large as 3 and 1 .

But here his observations startle him. Everything in 5 is familiar to him, including his own house which had been built on shell i! The correspondence is perfect in every detail; the house is exactly as he had left it. Yet he is quite certain that he is separated from surface 1 by the intervening shells. If he is to retain Euclidean geometry he must conclude that there are two identical worlds; every event on surface 1 is paralleled by an identical event on 5 . . 


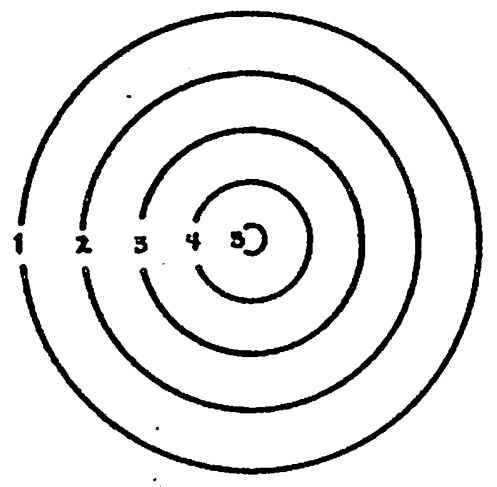

\section{(figure 1): Concentric Spherical Shells}

(to be conceived 3-dimensionaliy; the circles being cross sections of spherical shells in the plane of the drawing)

Figure 1 was drawn to represent the shells on a Euclidean interpretation. An alternative interpretation of these bizarre experiences would be to adopt the geometry of the torus (figure 2). On this interpretation our explorer starts at the shell whose cross section is appropriately labeled "1." lle travels "inward" toward the "hole" where the cross section of the second shell is labeled "2," continues traveling around the torus (his path of travel being represented by the dotted line) till he is on the "underneath" side at shell 3; naturally 3 measures larger than 2 and equal to 1 . Shell

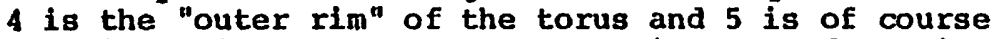
identical with 1, our explorer having come full circle.

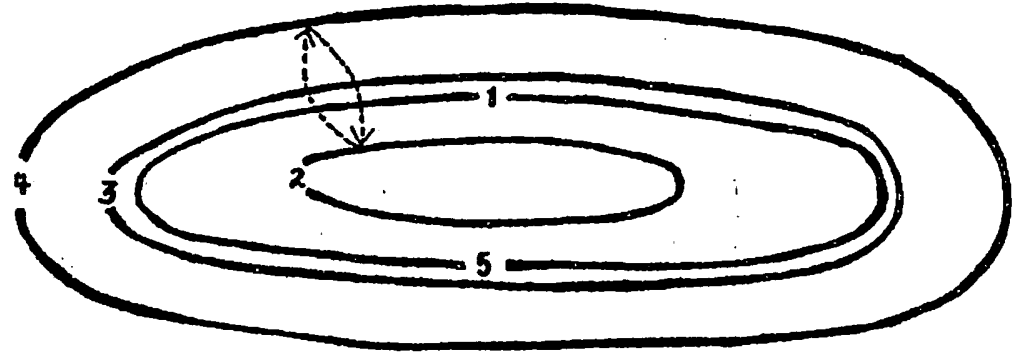

(figure 2): A Torus

Reichenbach has presented us with a hypothetical situation in which even positing a universal force 
(responsible for the contractions of the measuring rod) would not suffice to allow us to retain Euclidean geometry. In addition, a causal anomaly occurs; there is a spatial periodicity of all events la kind of preestablished harmony). Thus if our description in Euclidean geometry is to be topologically equivalent to the description in the Non-Euclidean geometry of the torus, we must, in Reichenbach's notation, write $G_{0}+F+A$ and not just $G_{0}+F$ (where $G_{0}$ is Euclidean geometry, $F$ is the universal field of force, and $A$ is the causal anomaly).

The causal anomaly manifests itself because the torus and the plane have different holistic properties (they are topologically different surfaces). While any two surfaces can be mapped one upon the other (as by the projection of shadows by light rays), it is only for surfaces with the same holistic properties that it is possible to carry through this transformation uniquely and continuously in all points. Here I use "uniquely" with Reichenbach to mean: "one and only one point of one surface corresponds to a given point of the other surface, and vice-versa"; i.e. there is a one-to-one function from the points of one surface onto the points of the other. "Continuously means: neighborhood relations in infinitesimal domains are preserved; no tearing of the surface or shifting of relative positions of points occur at any place" (Reichenbach, p. 59). Small regions of topologically different surfaces can be mapped thusly, but no such transformation is possible for the whole surface. It is this violation of continuity and/or uniqueness which corresponds in the physical interpretation to a causal anomaly.

4. The Disappearance of Universal Forces and the Elimination of Causal Anomalies. on the one hand Reichenbach believes that "the question concerning the structure of space" will not be meaningful unless universal forces are set equal to zero; "only then," he says, "is the problem of geometry uniquely determined" (Reichenbach, p. 27; cf. also Carnap's remarks in his "Introductory Remarks to the English Edition" of The Philosophy of space and Time, p. vil). On the other hand, Reichenbach says that, "the topological character of space . . . is determined only if we add the postulate of the disappearance of causal anomalies" (Reichenbach, p. 279; cf. also p. 80). Propounding both theses at once he says,

The metric of a space becomes an empirical fact only after the postulate of the disappearance of universal forces is introduced. Similarly, the topology of space becomes an empirical fact only 
if we add the postulate of the principle of action by contact (Reichenbach, p. 279).

Reichenbach would have us set both the universal field of force $F$ and the causal anomaly $A$ equal to zero. Unfortunately this is not always possible--consider another hypothetical situation analogous to Reichenbach's own:

This time our explorer measures shell 2 to be smaller than 1,3 smaller than 2,4 smaller still, and 5 even smaller. Upon arriving in shell 5 however, his experiences are even more bizarre than before. Not only is there a duplication of everything that he left in shell 1 but, since shell 5 is smaller, everything else is correspondingly smaller; he is a giant in a world identical to the one he just left. Upon returning to shell 1 , his fiends tell him of a similar giant who visited in his absence and finally left looking quite dumbfounded. Wandering off in the other direction the shells get increasingly larger till, in the fifth shell, he is a dwarf in a world exactly like the other two.

If he is to retain Euclidean geometry our explorer must assume that there is a series of identical worlds which get progressively smaller as you proceed in one direction and progressively larger in the other. No universal forces have been posited, yet a severe causal anomaly occurs. In other words, we have normalized $F=0$ but cannot, as Reichenbach would have us do, accept "normal" causality.

If we set A equal to zero however, we will require $F \neq 0$. If we choose to accept toroid geometry and the identity of these worlds, we will have to assume the presence of universal forces which increase the size of our explorer and his measuring rod when he goes from shell 2 to 3 and from 3 to 4 , but decrease his size when he goes from 4 to 3 and from 3 to 2 . Thus

$$
\mathbf{G}_{\mathbf{O}}+\mathbf{A}=\mathbf{G}+\mathbf{F}
$$

In this hypothetical situation we can, by the choice of geometry, set either $F$ or A equal to zero, but not both. Thus the example squeezes Reichenbach between his attempt to set $F$ equal to zero and his attempt to have $A$ equal to zero. He cannot object that such a state of the universe cannot exist; for we have started from the perceptions of an observer and, in Reichenbach's own words, "No a priori postulate can exclude the possibility that some person may at some time have certain perceptions. Only the interpretation of such perceptions is 
controversial" (Reichenbach, p. 77). If nothing else, this casts serious doubt on the universal acceptability of Reichenbach's twin "methodological" postulates of the disappearance of universal forces and the elimination of causal anomalies. If Reichenbach wants to maintain that we must set $F$ equal to zero, he cannot also require us to set $A$ equal to zero. If he chooses instead to go with $A$ having always to equal zero, he cannot require us to set $F=0$. None of this of course precludes him from insisting either that $F$ must always equal zero or that $A$ must always equal zero; it does however preclude him from doing both, and in so doing, casts doubt on the ultimacy of either. 4

"Causal anomaly" is a relative term; an anomaly under one set of causal laws may be a perfectly normal causal relation under a different set. For this reason it may be more fellcitous to break with Reichenbach and speak merely of differing sets of causal laws. The system we have been calling "normal" causality (with Reichenbach) is just another system of causality, there is no reason to believe that it should be uniquely picked out by stipulating that $F=0$. Thus instead of Reichenbach's claim that we have to accept "normal" causality for topology to be an empirical matter, we can say that the system of causality needs only to be specified; different topologies require different sets of causal laws.

5. Connections between Geometry and Causation. In Reichenbach's hypothetical example, as in my own, if the explorer decides to retain the principle of action by contact and refuses to admit any kind of preestablished harmony, then given his experiences he cannot consistently ascribe Euclidean geometry to physical space. Conversely, if he chooses to retain Euclidean geometry at all costs, his experiences will preclude him from adopting the traditional system of causal laws. Either alternative would be open to him and his choice could be fairly arbitrary, but he could not, so to speak. "have his cake and eat it too": he could not choose to retain both Euclidean geometry and "normal"

None of this shows that the principle of action by contact is, in fact, incompatible with Euclidean geometry; the question is an empirical one. A given geometry or vice-versa. It is on ly in conjunction with empirical experience that beliefs about causality have ramifications for the geometry of physical space. Similarly. unless concatinated with experience, geometry does not tell us anything about causation. But given experiences 
such as we have described, adhering to a set of causal laws will limit our freedom when assigning a geometry to physical space, and adhering to a geometry will curtail our freedom in formulating "laws" of causality.

Just how thorough-going are these connections between geometry and causation? Does a set of causal laws pick out a unique geometry for physical space? vice-versa? There is of course a rather trivial sense in which we can answer these questions in the negative: obvious ly our experiences can be scant or irrelevant and there is surely a half-way house where our experiences would suffice to establish some relationships between geometry and causation, but would simply be insufficient for a unique prescription. We are thereby enticed to move on to the more penetrating question as to whether a system of causal laws, in conjunction with unlimited experience, will pick out a unique geometry for physícal space, or vice-versa.

This question is not so easy to answer, but we can begin by recalling that differing sets of causal laws are required whenever we shift between geometries with different holistic properties. Any two geometries with the same holistic properties can be rendered equivalent by the addition of universal forces. Does this mean that topologically equivalent geometries will never require different causal laws? Not necessarily; universal forces may themselves sometimes constitute a type of causal anomaly. We must investigate the causal status of universal forces. To this end it will be instructive to consider gravitation, although it is not purely a universal force. 6

Bertrand Russell, for one, rejoiced in Einstein's treatment of gravitation. With the adoption of nonEuclidean geometry, he observed, we no longer need to assume that the sun somehow "makes" the earth travel in an ellipse about $1 t$; the earth simply travels along the natural geodesic prescribed by the geometry: no "forces" or "little pushes" are required to keep it on its orbit.7 It is not that we have excused ourselves from giving a causal explanation of the orbit--we are using geometrical considerations as our physical explanation, not rejecting the need for one. The geometry of the universe serves as our "cause."

In such a case, the change of geometry and the resultant postulation of universal forces entails a corresponding change in the causal explanation of certain phenomena. It may well be that a change in geometry always entails a change in the explanation of some such phenomenon. Still, the point of the causal anomaly was 
that it represented a change in causal 1aws; changing our causal explanations of certain phenomena is not the same thing as changing our system of causal laws. We seek to know whether changing from one geometry to another with the same holistic properties involves a change in causal laws.

Part of our difficulties here stem from our ignorance about what actually constitutes a change in causal laws. I suggest that this much is certain: a change in causal laws is involved whenever the explanation under one of the geometries requires a principle not used to explain any phenomena whatsoever under the other geometry. Thus if one geometry could support explanations of all phenomena without invoking action at a distance, but the other geometry could not (as in Reichenbach's hypothetical example), we would say that a change in causal laws was required.

A further source of difficulty may seem to lie in our agreement to allow unlimited experience; how are we to know what unlimited experience would turn up? Fortunately, we are not tied to the experiences of the "real" world; we have recourse to hypothetical situations. Thus we can assume that gravitation is a universal force, pure and simple, and not be bothered about its differential effects (there simply won't be any in our hypothetical worlds). If we assume that, given enough time, physics will
discover some gravitons or demonstrate the existence of the aether, we may conclude that the change in geometry analogous to that proposed by Einstein will not necessitate a change in causal laws; no differentiating principle of causation would be needed. If on the other hand, we assume that even unlimited experience will not turn up such substances, the change in geometry would entail a change in causal laws. Adhering to Euclidean geometry would preclude us from adopting a system of causation which did not sanction action at a distance; the alternative geometry, however, would not require
the use of this principle.

So we have one case in which a change between geometries with the same holistic properites requires a change in the system of causal explanation and one case in which it does not. We have already seen that changing between geometries with different holistic properties requires a change in the causal laws. The interesting point about discovering gravitons or aether is that Euclidean geometry would not have to use actionat-a-distance to explain gravitational forces and hence could employ the same set of causal laws as does the 
does not always necessitate a change in the system of causal laws.

Now we have left only to investigate whether a change in causal laws will always entail a change in geometry; given the same unlimited experiences, is more than one system of causal laws compatible with any one geometry? It is important to notice the qualification "given the same unlimited experiences"; in our contact under one set of experiences and compatible with action at a distance under another set. But we did not thereby show that, given only one set of experiences, we can make a change in our system of causal laws without having to change our geometry also.

As one might expect, the real difficulty arises in trying to find two differing systems of causal laws which cannot be decided between on the basis of experience. To this end, the philosophy of Bishop Berkeley will prove to be a blessing. For Berkeley, to exist is to be perceived (esse est percipi). Leaving. aside Berkeley's divine perceiver. objects continually pop in and out of existence; I can make this peiece of paper cease to exist and then recreate it again simply by turning my head for a few seconds. Obviously there must be some sort of causal connection between the observer and the object observed. In fact Berkeley's system was intended as an alternative to that system which explains the existence of physical objects by some kind of underlying substratum or substance (John Locke's system in particular). Naturally. Berkeley's system is at variance with present day physics; it represents a different set of causal laws.

Berkeley 's system was never well-accepted; even at the outset it was thought to be disproved simply by kicking a stone. But the system is not so easily disproved; to show that it is mistaken one would have to demonstrate the existence of a physical object at a time when it was not perceived by anyone.8 His system has the distinctive feature that it cannot be rejected simply by using sensory experience; if we are to reject the system, it must be on some other grounds.

But the geometry of physical space will not provide such a basis; there is nothing about causing objects to exist by perceiving them which requires us to give up Euclidean, or any other geometry for that matter (i.f there were, you can be assured that someone would have advanced it as an argument against Berkeley). The conclusion is obvious: even given the same set of unlimited experiences there is nothing about changing to the causal laws dictated by Berkeley's system which would require us to change the geometry we assign to physical space; a 
change in causal laws will not always entail a change in geometry.

6. Summary. In addition to showing that Reichenbach is mistaken in wanting to set both $F$ and $A$ equal to zero, we have established (i) that a change in geometry can compel a corresponding change in our system of causal laws, 9 (ii) that a change in geometry can take place without entailing a change in the system of causal laws, 10 (iii) that a change in causal laws can require a change in the geometry we assign to physical space, 11 and (iv) that a change in causal laws can be made without requiring a change in geometry.12 conjoining these four theses we may say that, though there are times when a change in causal laws will entail a change in geometry (and vice-versa), even in conjunction with unlimited experience, a system of causal laws will not necessarily suffice to pick out a unigue geometry (or vice-versa). The connections between geometry and causation are close indeed, but not close enough for a unique prescription.

University of Illinois at Chicago Circle 
NOTES

${ }^{1}$ cf. Hans Reichenbach, The Philosophy of Space and Time, Eng. ed. (New York: Dover, 1958), pp. 80, 279.

2 It may be helpful to note that the "actual" geometry $G^{\circ}$, for which $F$ has been set equal to zero, is just that geometry which results when we assume our measuring rods to be rigid.

$3_{\text {The force is universal rather than differential }}$ since it affects all substances in exactly the same way and cannot be shielded against; it is a literal force which is said to destroy coincidences since it is operative on shell 3 , but not on shells 1 and 2 .

4ncidently, we might still go along with him about setting both equal to zero if possible and use some other criteria (e.g. overall simplicity of the system) to decide which, if either, to set equal to zero if a situation such as we have described should ever arise.

5 The alternative of disbelieving the sensory experiences is ruled out because the experiences are quite reproducible and the measurements and observations can be repeated as often as one should care to go through the procedure.

${ }^{6}$ Gravitation is universal in that it cannot be shielded against and, in some respects, affects all substances in the exact same way $(\mathrm{e} . \mathrm{g}$. the rate of acceleration of a falling body does not vary with the chemical composition of that body). But the force is differential in certain of its other effects (e.g. a wooden bookshelf will sag more under the influence of gravity than a steel one) (cf. Grunbaulm pp. 91-92).

7Bertrand Russell, An Outline of Philosophy (1927;

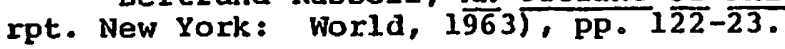

${ }^{8}$ Things become further complicated if we introduce the divine perceiver since even this demonstration would fail to disprove the theory: I have left him out for two reasons: (1) unlimited experience should perhaps turn up the existence of such a god, and (2) leaving him out simply makes for a sharper distinction in the systems of causal laws. 
9 This is established for geometries with different holistic properties by Reichenbach's hypothetical example and, for geometries with the same holistic properties. by my reflections on gravitation.

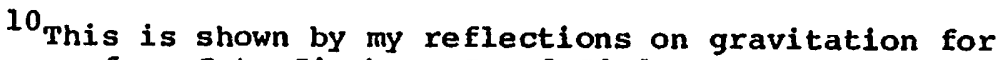
a change from $G$ to $G^{\prime}$ where $G$ and $G^{\prime}$ do not have different holistic properties.

$11_{\text {This is established by Reichenbach's hypothetical }}$ example. Given the experiences of our explorer, if his causal laws preclude pre-established harmony of any sort, he cannot consistently ascribe Euclidean geometry to physical space; which is not to say that his causal. laws have uniquely picked out the replacement geometry, though they have presumably ruled out all geometries topologically similar to the Euclidean planar geometry.

12 This is what we just showed using Berkeley's alternative system. 